\title{
Intersections
}

Canadian Journal of Music

Revue canadienne de musique

\section{Annegret Fauser. Musical Encounters at the 1889 Paris World's Fair. Eastman Studies in Music, vol. 32. Rochester : University of Rochester Press, 2005. xviii, 391 p. ISBN 1-58046-185-9 \\ (couverture rigide)}

\section{Marie-Hélène Benoit-Otis}

Volume 27, numéro 1, 2006

URI : https://id.erudit.org/iderudit/1013165ar

DOI : https://doi.org/10.7202/1013165ar

Aller au sommaire du numéro

Éditeur(s)

Canadian University Music Society / Société de musique des universités canadiennes

ISSN

1911-0146 (imprimé)

1918-512X (numérique)

Découvrir la revue

Citer ce compte rendu

Benoit-Otis, M.-H. (2006). Compte rendu de [Annegret Fauser. Musical

Encounters at the 1889 Paris World's Fair. Eastman Studies in Music, vol. 32.

Rochester : University of Rochester Press, 2005. xviii, 391 p. ISBN 1-58046-185-9

(couverture rigide)]. Intersections, 27(1), 106-111.

https://doi.org/10.7202/1013165ar

All Rights Reserved (C) Canadian University Music Society / Société de musique des universités canadiennes, 2007
Intersections CANADAN TOURAAL OP NUST
Ce document est protégé par la loi sur le droit d'auteur. L’utilisation des services d'Érudit (y compris la reproduction) est assujettie à sa politique d'utilisation que vous pouvez consulter en ligne.

https://apropos.erudit.org/fr/usagers/politique-dutilisation/ 
purpose was to create an Upper Austrian competitor to the Salzburger Festspiele that had so effectively capitalized on the legacy of Mozart. The moral of the story is that we forget how easily the boundaries between innocuous entertainment and emotional manipulation of large masses of people become blurred.

Beyond its academic merits, Music, Theatre and Politics in Germany is one of those books that invite the reader and theatre enthusiast to explore new repertories. Though the standard works of the period from Wagner through Schoenberg, Berg and Weill are well known, the authors certainly encourage the exploration of lesser known works like Weber's Euryanthe, Schumann's Genoveva, and the operas of Franz Schreker, Ernst Toch, Ernst Krenek and Karl Amadeus Hartmann. Even Hindemith's operas are really not as well known as they should be. Part of the problem will always be that especially the works in a more modernist style are actually competing with the limited resources that opera companies have available for works by the living composers of today. Nevertheless I would hope that, perhaps through concert performances, some of these neglected works can be recorded, thereby increasing the chances of their return to the stage.

J. Alexander Colpa

Annegret Fauser. Musical Encounters at the 1889 Paris World's Fair. Eastman Studies in Music, vol. 32. Rochester : University of Rochester Press, 2005. xviii, 391 p. ISBN 1-58046-185-9 (couverture rigide).

Bien qu'elle occupe une place emblématique dans l'histoire de la musique, l'Exposition universelle parisienne de 1889 a rarement été étudiée pour elle-même. En effet, c'est surtout sous l'angle de la réception, en particulier par les principaux compositeurs français de l'époque, que les musicologues et les historiens se sont jusquà maintenant intéressés à la musique présentée à l'Exposition; on pense en particulier à la rencontre de Debussy avec le gamelan javanais, qui allait devenir une source d'inspiration si importante pour le compositeur ${ }^{1}$.

C'est là l'un des sujets que reconsidère Annegret Fauser dans son ouvrage sur la présence de la musique à l'Exposition universelle parisienne de 1889. Après une thèse de doctorat sur le genre de la mélodie française avec orchestre de 1870 à 1920 (Rheinische Friedrich-Wilhelms-Universität, Bonn, 1992; parution chez Laaber en 1994), l'auteur a publié de nombreux articles sur la musique française des $\mathrm{XIX}^{\mathrm{e}}$ et $\mathrm{XX}^{\mathrm{e}}$ siècles. Elle a également réuni un dossier de presse de la création d'Esclarmonde de Massenet (Fauser 2001), et, en collaboration avec Manuela Schwartz, édité un ouvrage collectif majeur sur Wagner et le wagnérisme en France (Fauser et Schwartz 1999). Dans Musical Encounters at the 1889 Paris World's Fair, elle relève un défi de taille : reconstituer le monde sonore de

1 Cet épisode de la biographie de Debussy est relaté, entre autres, dans Lockspeiser 1966, p. 113 116, et dans Lesure 2003, p. 103-105. À propos de l'influence du gamelan sur la musique de Debussy, voir notamment Arndt 1993 et Howat 1994; dans son sous-chapitre sur Debussy (p. 195-206), Fauser énumère de nombreuses autres références sur ce sujet (voir en particulier p. 198). 
l'Exposition universelle (en s'intéressant tant à la musique que, plus généralement, au son, omniprésent lors de lévénement), à partir des documents muets qui, comme elle le souligne avec justesse, constituent le matériau de base de l'historien².

Pour ce faire, Fauser procède à un minutieux dépouillement de très nombreuses sources primaires : comptes rendus et programmes de concerts et de spectacles, ouvrages sur la musique de la fin du XIX ${ }^{e}$ siècle, correspondance de compositeurs, guides touristiques à l'intention des visiteurs de l'Exposition, etc. En replaçant soigneusement ces documents dans leur contexte sociopolitique et en les faisant dialoguer avec les quelques sources proprement musicales disponibles (partitions, transcriptions de musiques exotiques par des musiciens français), Fauser parvient à en faire émerger une image remarquablement claire de la musique présentée à l'Exposition, au-delà de sa seule réception, traitée ici comme un moyen plutôt que comme une fin en soi.

Les six chapitres de louvrage sont traversés par deux fils conducteurs, formulés dès l'introduction : le nationalisme (en particulier français) et la question de l'authenticité ${ }^{3}$, directement liée à celle du rapport à l'Autre. Fauser montre comment, dans le contexte d'une Exposition présentée à l'occasion du centenaire de la Révolution française, la musique devient le vecteur d'une véritable célébration nationale et républicaine ayant pour objectif de démontrer la grandeur de la France, aussi bien en tant que puissance coloniale quen tant que nation culturelle, vue comme un équivalent moderne de la Rome antique. Cette dynamique nationaliste est favorisée par le fait qu'à l'Exposition, toutes les musiques sont inscrites dans un contexte national, que ce soit au sein des pavillons consacrés aux différents pays ou dans le cadre de concerts thématiques. Par ailleurs, cette disposition par pays soulève la question de l'authenticité, essentielle dans la réception des musiques non occidentales présentées à l'Exposition : confrontés pour la première fois directement avec la musique des Autres, sans le filtre du pseudo-exotisme pratiqué par les musiciens français ${ }^{4}$, les visiteurs tiennent en effet à ce que cette musique corresponde réellement aux traditions du groupe qui l'interprète.

2 "This gap between historic sound and documentary trace leads directly into the heart of one of the most fascinating issues of historiography : the dichotomy of sensual immediacy and, more specifically, of our listening imagination on the one hand, and of the silence of historical documents and scholarly discourse on the other. All too rarely do we question the business of history as one that engages generally only with silent traces of historic events ", p. 10-11. Dans un récent article, Fauser présente un important complément à la réflexion historiographique menée en filigrane de Musical Encounters at the 1889 Paris World's Fair (voir Fauser 2006).

3 Fauser emploie le terme d'authenticité dans trois sens analogues, mais légèrement différents : d'abord comme un synonyme de véritable, d’origine (genuine), par opposition à déventuelles imitations ou contrefaçons de la musique d'une région ou d'une époque donnée; ensuite dans le sens plus étroit de l'exactitude de l'interprétation de ces musiques; enfin, en référence à la fidélité de la reproduction sonore des nouvelles technologies, comme le phonographe (voir p. 13).

4 Dans des œuvres comme Lakmé, de Delibes, ou La princesse jaune, de Saint-Saëns. À propos de lexotisme, Fauser démontre (p. 139-146) qu'au XIXe siècle, les compositeurs français désireux dévoquer un univers extra-européen ont recours à des stéréotypes pseudo exotiques (comme l'emploi de secondes augmentées, d'échelles pentatoniques ou de rythmes de danse typiques comme celui de la habañera) et citent à loccasion des extraits de musiques originales collectées par des voyageurs, le tout sans outrepasser les frontières du langage tonal et des formes traditionnelles. 
Les trois premiers chapitres de l'ouvrage de Fauser portent sur la représentation de la musique savante occidentale à l'Exposition, les quatrième et cinquième chapitres sur les musiques non occidentales et folkloriques, et le dernier chapitre traite des nouveautés technologiques reliées au son, exposées au pavillon de la Société générale des téléphones et à la Galerie des machines. Dans le premier chapitre, Fauser montre comment, dans le cadre de l'Exposition, la musique savante est mise en scène de manière à souligner la grandeur passée et présente de la France. Cela s'exprime dans les expositions d'instruments modernes, qui exaltent la facture française de pianos, ainsi que dans différentes séries de concerts de musique instrumentale et vocale présentés, pour l'essentiel, au Palais du Trocadéro, la salle de concert officielle de l'Exposition. Ainsi, une série de cinq concerts intitulée "Auditions officielles de musique française " permet de présenter les cinq grands orchestres parisien $s^{5}$, en plus détablir un canon de chef-d'œuvres français à saveur nationaliste, par le biais d'une rétrospective de la musique composée en France au cours des cent ans écoulés depuis la Révolution. Des concerts de musique ancienne sur instruments d'époque ont pour objectif de retrouver les bases historiques sur lesquelles fonder les nouveaux développements de la musique française; une série de concerts nationaux présentant la musique de différents pays (Russie, Finlande, États-Unis, Norvège, Espagne et Belgique) fournit loccasion de comparer la production musicale étrangère avec celle de la France. En bout de ligne, conclut Fauser avec une subtile ironie, mais sans s'aventurer à émettre quelque jugement que ce soit sur cet énergique nationalisme, la représentation de la musique à l'Exposition universelle a pour but de "prendre le monde entier à témoin du triomphe de l'Ars gallica " ${ }^{6}$ (p. 58).

Le deuxième chapitre traite de la musique de scène, opéra et ballet, créée ou représentée à l'Exposition. Il s'agit, en premier lieu, d'Esclarmonde de Massenet, demeuré la seule création d'opéra de l'Exposition suite au report de la première d'Ascanio de Saint-Saëns. Créé à l'Opéra-Comique le jour du dévoilement de la tour Eiffel, lopéra de Massenet suscite une admiration comparable, notamment en raison de la performance de la soprano américaine Sybil Sanderson, qui interprète le rôle-titre à grand renfort de "notes-Eiffel " (p. 63), ainsi que de la mise en scène à grand déploiement, avec éclairage électrique. Aussi bien le livret que la musique d'Esclarmonde donnent lieu à des discussions à saveur nationaliste, dont l'enjeu est essentiellement de savoir si l'opéra de Massenet peut ouvrir une nouvelle voie à l'opéra français, au-delà de l'influence écrasante de Wagner ${ }^{7}$. Des questions similaires sur l'avenir d'un genre traditionnellement français marquent la création, à l'Opéra, du ballet $\mathrm{La}$ tempête d'Ambroise Thomas, librement inspiré de Shakespeare et dont les critiques se demandent s'il saura renouveler le ballet par un retour aux « saines traditions de la chorégraphie » (p. 99).

5 Il sagit des orchestres des Concerts Lamoureux, des Concerts Colonne, du Conservatoire, de l'Opéra et de l'Opéra-Comique.

6 "The official musical setup of the Exposition Universelle may not have been as extensive as the one ceded to visual arts, but it ensured that the world witnessed (potentially) the triumph of Ars Gallica. " Traduction libre.

7 Dans louvrage de Fauser, Esclarmonde est surtout traité du point de vue de sa réception dans le cadre de l'Exposition universelle. Ainsi, ce sous-chapitre adopte une position complémentaire par rapport à l'analyse approfondie de l'opéra déjà réalisée par Steven Huebner (1999, p. 73-81 et 82-101). 
Le troisième chapitre est consacré à la création de l'Ode triomphale en l'honneur du centenaire de 1789, d'Augusta Holmès, spectacle à grand déploiement qui constitue le point culminant des célébrations du centenaire de la Révolution. Fauser reconstruit la représentation de cette œuvre chorale d'envergure et en livre une brillante analyse, centrée sur un réseau intertextuel dans lequel figurent notamment des œuvres révolutionnaires de François-Joseph Gossec. Elle se penche ensuite sur la réception de l'Ode triomphale, plus ou moins favorable selon les opinions politiques de chacun des commentateurs, mais dans laquelle un thème est abordé par tous : le fait que le compositeur soit une femme. Si la féminité d'Holmès entre en conflit avec le caractère de "virilité " attribué à une œuvre musicale de grandes dimensions, elle cadre bien avec le contexte politique du centenaire de la Révolution : Fauser démontre en effet que Holmès peut facilement être identifiée à la figure de Marianne, d’où le titre de ce chapitre, « La muse de la République ".

Dans le quatrième chapitre, Fauser aborde les musiques extrême-orientales présentées à l'Exposition, en particulier le kampong javanais et le théâtre annamite. Elle prend d'abord soin d'établir le contexte dans lequel ces musiques sont entendues en 1889, en traçant un portrait, d'une part, de l'exotisme musical pratiqué par les compositeurs français du XIX siècle, et d'autre part, de l'histoire de la musique à saveur téléologique et ethnocentrique (pour ne pas dire carrément raciste) qui domine à cette époque. Cette toile de fond lui permet de faire ressortir clairement à quel point des représentations comme celles du gamelan javanais et du théâtre annamite constituent une nouveauté absolue pour le public, même musicien. Fauser va cependant au-delà de la seule histoire de la réception, et propose une reconstitution des spectacles offerts par les troupes javanaise et vietnamienne, fournissant un maximum d'informations sur les acteurs de ces représentations dont on ne savait presque rien jusquà maintenant.

Elle procède de façon semblable dans le cinquième chapitre, consacré aux musiques du Moyen-Orient et de l'Afrique, ainsi qu'au folklore européen. Les musiques moyen-orientales étant insuffisamment traitées par des commentateurs peu au fait des traditions musicales de cette partie du monde, Fauser a recours à des documents d'archives pour identifier instruments et ensembles. La musique africaine, présentée dans des cafés-concerts et dans le cadre d'expositions vivantes de la section coloniale, est évoquée de façon encore plus sommaire dans les sources consultées par Fauser, et considérée, dans le meilleur des cas, comme une étape primitive du développement de la musique. La musique folklorique, enfin, jouit d'un statut relativement élevé en raison du caractère de " voix authentique du peuple » qu'on lui attribue; elle est donc relativement bien documentée. Fauser montre que dans toutes ces musiques " autres " ${ }^{8}$, mais qui, contrairement à celles de Java et du Vietnam, ne sont pas complètement nouvelles pour le public français, la question de l'authenticité prend une importance capitale. Ainsi, la présence d'un piano dans l'ensemble tunisien provoque une réaction pour le moins mitigée (p. 237-238), et le fait que l'ensemble folklorique 
russe ait engagé des musiciens français est dénoncé dans la presse comme une véritable fraude (p. 268).

Le sixième et dernier chapitre porte sur les technologies musicales dont les derniers développements sont présentés à l'Exposition : le phonographe d'Edison, exposé à la Galerie des machines, et la retransmission d’opéras par téléphone, une technologie permettant aux visiteurs du pavillon de la Société générale des téléphones découter à distance et en direct, au moyen de tubes acoustiques, les représentations de l'Opéra et de l'Opéra-comique. Dans ce chapitre, Fauser décrit avec minutie les installations sonores de l'Exposition et l'effet que ces dernières produisent sur les visiteurs. Elle livre également une fascinante discussion des relations entre les modes découte de la musique à la fin du $\mathrm{XIX}^{\mathrm{e}}$ siècle et l'avènement des nouvelles technologies de transmission du son : elle situe l'audition isolée à l'aide de tubes acoustiques dans le prolongement du mode d'écoute intériorisé préconisé par les Romantiques, dans lequel lauditeur concentre son attention sur une méditation intérieure coupée du monde (en particulier du monde visuel) qui l'entoure. Elle conclut en soulignant les limites des technologies sonores de 1889 , en particulier du phonographe, qui représente une promesse de réalisations futures, une « musique de l'avenir plutôt que du présent $"^{9}$ (p. 309).

Dans l'ensemble, louvrage de Fauser est une remarquable réussite. Très bien structuré et rédigé dans un style clair et agréable, accessible aussi bien à l'amateur éclairé qu'au spécialiste de la musique de cette période, il constitue une lecture d'autant plus passionnante qu'il contient une impressionnante iconographie (plus de 60 illustrations) qui rend encore plus vivante la reconstitution de l'auteur. Les annexes, dans lesquelles sont répertoriés programmes de concerts et représentations d'opéra, contribuent à faire de ce livre un véritable ouvrage de référence. Mais au-delà de la nouveauté du sujet et d'une méthodologie irréprochable à tous points de vue, c'est la profondeur de la réflexion qui rend cet ouvrage si essentiel : loin de se contenter de réaliser une étude historique basée sur la réception, Fauser questionne les prémisses méthodologiques de sa discipline et explore les implications politiques, sociales et esthétiques de l'univers quelle parvient à recréer. Elle remet également en question plus d'une opinion communément admise, et n'hésite pas à reconsidérer sous un nouveau jour des sujets sur lesquels beaucoup a déjà été écrit.

C'est le cas notamment de la découverte du gamelan javanais par Debussy, évoquée au début de cette recension. Fauser attire l'attention sur les limites de la littérature secondaire déjà publiée sur ce sujet, dans laquelle il est question uniquement de l'influence de l'univers sonore de la musique javanaise sur les œuvres pour piano et pour orchestre de Debussy. Elle démontre que l'aspect visuel et dramatique, aussi bien du théâtre annamite que du kampong javanais, ont constitué une source d'inspiration essentielle pour Debussy, notamment dans la genèse de son opéra Pelléas et Mélisande. En outre, elle propose de réinterpréter lexotisme musical de Debussy comme une forme d'appropriation inscrite dans

9 "Thus the Edison exhibit of the phonograph was, in the end, presenting a music of the future rather than of the present, a promise of things to come. " Notre traduction. 
les traditions musicales françaises des années 1890 , plutôt que comme une rupture menant à la modernité ${ }^{10}$ (p. 205). Cette vision, nouvelle et convaincante, constituerait un excellent point de départ pour des travaux futurs.

\section{RÉFÉRENCES}

Arndt, Jürgen. 1993. Der Einfluß der javanischen Gamelan-Musik auf Kompositionen von Claude Debussy. Frankfurt : Peter Lang.

Fauser, Annegret. 1994. Der Orchestergesang in Frankreich zwischen 1870 und 1920. Freiburger Beiträge zur Musikwissenschaft, vol. 2. Laaber : LaaberVerlag.

dir. 2001. Dossier de presse parisienne : Jules Massenet, "Esclarmonde " (1889). Heilbronn : Musik-Edition Lucie Galland. 2006. " Histoires interrompues : Raconter l'histoire de la musique moderne en France ». Dans Musique et modernité en France, sous la dir. de Sylvain Caron, François de Médicis et Michel Duchesneau, p. 19-50. Montréal : Presses de l'Université de Montréal.

Fauser, Annegret, et Manuela Schwartz, dir. 1999. Von Wagner zum Wagnérisme: Musik, Literatur, Kunst, Politik. Transfer : Die deutsch-französische Kulturbibliothek, vol. 12. Leipzig : Leipziger Universitäts-Verlag.

Howat, Roy. 1994. "Debussy and the Orient ". Dans Recovering the Orient : Artists, Scholars, Appropriations, sous la dir. de Andrew Gerstle et Anthony Milner, p. 45-81. Chur : Harwood Academic Publishers.

Huebner, Steven. 1999. French Opera at the Fin de Siècle : Wagnerism, Nationalism, and Style. Oxford : Oxford University Press.

Lesure, François. 2003. Claude Debussy: Biographie critique suivie du catalogue de l'ouvre. Paris : Fayard.

Lockspeiser, Edward. 1966 [1962]. Debussy : His Life and Mind, vol. $1: 1862-$ 1902. Londres : Cassel.

MARIE-HÉLÈNe BeNOIT-OTIS

10 « Rather, I would suggest that we reinterpret his [Debussy's] fascination with the spectacle of the kampong javanais and the Théatre Annamite as an encounter with alterity not as an agent of rupture as so often posited by the modernist construction of Debussy but as a form of appropriation firmly inscribed in the tradition of French music in the 1890s. " Notre traduction. 